\title{
Manifestations of magnetic vortices in the equation of state of a Yang-Mills plasma
}

\author{
M.N. Chernodub, ${ }^{1}$ Atsushi Nakamura, ${ }^{2}$ and V.I. Zakharov ${ }^{3,1}$ \\ ${ }^{1}$ Institute of Theoretical and Experimental Physics, \\ B.Cheremushkinskaya 25, Moscow, 117218, Russia \\ ${ }^{2}$ Research Institute for Information Science and Education, \\ Hiroshima University, Higashi-Hiroshima, 739-8527, Japan \\ ${ }^{3}$ Istituto Nazionale di Fisica Nucleare - Sezione di Pisa, \\ Dipartimento di Fisica Universita di Pisa, Largo Pontecorvo 3, 56127 Pisa, Italy
}

(Dated: July 31, 2007)

\begin{abstract}
The vacuum of Yang-Mills theory contains singular stringlike objects identified with center (magnetic) vortices. Percolation of magnetic vortices is known to be responsible for the color confinement in the low-temperature phase of the theory. In our work we study properties of the vortices at finite temperature using lattice simulations of $S U(2)$ gauge theory. We show that magnetic vortices provide a numerically large contribution to thermodynamic quantities of the gluon plasma in Yang-Mills theory. In particular, we observe that in the deconfinement phase at temperatures $T_{c}<T \lesssim 3 T_{c}$ the magnetic component of the gluon plasma produces a negative (ghostlike) contribution to the anomaly of the energy-momentum tensor. In the confinement phase the vortex contribution is positive. The thermodynamical significance of the magnetic objects allows us to suggest that the quark-gluon plasma may contain a developed network of magnetic flux tubes. The existence of the vortex network may lead to observable effects in the quark-gluon plasma because the chromomagnetic field of the vortices should scatter and drag quarks.
\end{abstract}

PACS numbers: 12.38.Aw, 25.75.Nq, 11.15.Tk

\section{INTRODUCTION}

Studies of properties of thermal plasma became a major development in QCD in recent years, for a review see, e.g. [1, 2]. Properties of the plasma are studied both directly, at RHIC and via lattice simulations. On the theoretical side, novel ideas, like AdS/CFT correspondence are being invoked [3], to say nothing of traditional approaches based on various quasiparticle models [4] and on field theory at finite temperature.

The traditional approach to the thermal plasma treats it, in zero approximation, as gas of free gluons and quarks and, then, takes into account perturbative corrections. An outcome of such calculations is a representation of the energy and pressure densities as perturbative series in the effective coupling constant $g^{2}(T)$ :

$$
\epsilon(T)=c_{\mathrm{SB}} T^{4} f_{\text {pert }}\left(g^{2}(T)\right),
$$

where $c_{\mathrm{SB}}$ is the Stefan-Boltzmann (SB) coefficient, proportional to the number of degrees of freedom, and the perturbative expansion, $f_{\text {pert }}\left(g^{2}(T)\right)$ is known explicitly up to terms of order $g^{6} \ln T$. Perturbative predictions for such global characteristics of the plasma as energy density turn to be in reasonable agreement with the data [2].

On the other hand, some particular properties of the plasma, such as viscosity [5], indicate that, in the zero approximation, plasma is to be considered rather as an ideal liquid than an ideal gas. There is no yet a coherent picture that would unify both perturbative and nonperturbative features of the QCD plasma.

It was speculated in Refs. [6, 7, 8] that there exists a magnetic component of the Yang-Mills plasma which at temperatures not much above the critical temperature
$T_{c}$ is crucial for the plasma properties. In Refs. 7, 8] constituents of the magnetic component are thought to be classical magnetic monopoles. In Ref. [6] the magnetic component is identified with so-called magnetic strings related to magnetic monopoles. The properties of the strings, or center vortices and their role in confinement have been discussed in the lattice community for more than a decade, for review and references see Ref. [9].

According to the vortex picture the quark confinement emerges due to spatial percolation of the magnetic vortex strings which lead to certain amount of disorder. The value of the Wilson loop changes by a center element of the gauge group if the magnetic vortex pierces the loop. Therefore, very large loops receive fluctuating contributions from the vortex ensembles. These fluctuations make the average value of the Wilson loop very small. One can show that the suppression of the loop follows an area law for very large loops $[9]$.

Magnetic monopoles are certain gluonic objects that are related to color confinement via the so-called dual superconductor mechanism [10]. The condensate of the monopoles - which is formed in the low-temperature phase - expels the chromoelectric field exhibiting a dual analogue of the Meissner effect. The chromoelectric field of quarks is squeezed into a dual analogue of the Abrikosov vortex leading to quark confinement (for a review see Ref. [11]).

The presence of both the magnetic monopoles and the magnetic vortices in the vacuum of Yang-Mills theory seem to be an indication of the existence of a more complicated genuine non-Abelian object. Indeed, Abelian monopoles and center vortices appear to be strongly correlated with each other 12]: almost all monopoles are 
sitting on top of vortices. In $S U(2)$ gauge theory the genuine object is a monopole-vortex chain [12]. In $S U(3)$ Yang-Mills theory one can expect existence of monopolevortex 3-nets: the junction of the three vortices may be viewed as a nexus [13] or/and as a center monopole [14]. An illustration of the chains and nets in a 3-dimensional timeslice is shown in Figure 1.

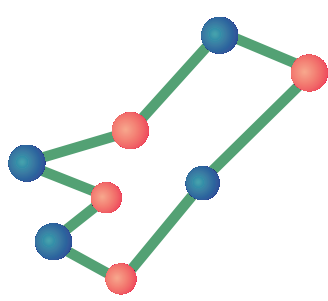

SU(2) chains

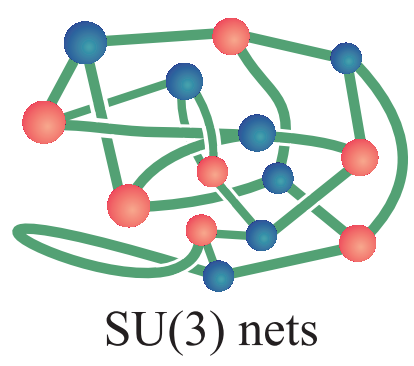

FIG. 1: (Color online) An illustration of the monopole-vortex chains and the monopole-vortex nets in $\mathrm{SU}(2)$ and $\mathrm{SU}(3)$ Yang-Mills theories, respectively.

The formation of these composite objects is essential for the self-consistent treatment of monopoles in the quark-gluon plasma [6]. Similar monopole-vortex chains were found in numerous (non-)supersymmetric nonAbelian gauge theories involving various Higgs fields [15, 16]. In particular, in Abelian gauge models with a compact gauge field, monopoles and vortices should be organized in chains/nets in order to be consistent with breaking of the confining electric string in specific representations of the gauge group [16]. A short overview of the monopoles and vortices in the Yang-Mills plasma can be found in Ref. [17].

In terms of the continuum theory, the magnetic strings are defined as (infinitely thin) surfaces that are closed in the vacuum state and can be open on an external 't Hooft line (the same as the standard, or "electric" strings can be open on the Wilson line). One can argue (for review and references see Ref. [18]), that the magnetic strings of the continuum theory can be identified with the center vortices of lattice studies. Moreover, it is also known that, indeed, properties of the vortices are changing drastically once temperature is increased above the critical value $T_{c}$ 9, 19, 20, 21, 22]. In particular, they become time oriented and this agrees with the assumption that the vortices become a component of the Yang-Mills thermal plasma [6].

Once the magnetic component of the plasma is identified with the magnetic vortices, further information on its properties can be obtained by direct numerical calculations on the lattice at finite temperature. In this paper, we report on results of lattice calculations of contribution of magnetic strings to the trace anomaly of energy-momentum tensor in the gluon plasma. The trace anomaly is intimately related to the equation of state, or energy density and pressure of the thermal Yang-Mills plasma. Preliminary results of our investigation were reported in Ref. 23]. Our lattice calculations refer to the case of pure $S U(2)$ gauge theory which is conceptually similar to a more realistic theory with three colors.

The structure of the paper is as follows. In Section II we discuss basic relations of Yang-Mills thermodynamics both in the continuum limit and on the lattice. In Section III we present our results for the thermodynamics of $S U(2)$ Yang-Mills theory on moderately-sized lattice. Although the numerical results on the $S U(2)$ thermodynamics are available in the literature [24] we repeat the calculations on lattices of particular geometries as these lattices are later used in Section IV] to calculate thermodynamical properties of magnetic vortices such as the thermal vortex density and contribution to the (trace) anomaly of the energy momentum tensor. A short summary of our results is given in Section $\nabla$ which is followed by a concluding Section.

\section{YANG-MILLS THERMODYNAMICS AND TRACE ANOMALY}

\section{A. Continuum thermodynamics}

The free energy of an $S U(N)$ gauge system

$$
F=-T \log \mathcal{Z}(T, V),
$$

is expressed via the partition function $\mathcal{Z}$ as follows:

$$
\mathcal{Z}=\int \mathrm{D} A \exp \left\{-\frac{1}{2 g^{2}} \operatorname{Tr} G_{\mu \nu}^{2}\right\}
$$

where $G_{\mu \nu}=G_{\mu \nu}^{a} t^{a}$ is the field strength tensor of the non-Abelian field $A$, and $t^{a}$ are the $S U(N)$ generators normalized in the standard way, $\operatorname{Tr} t^{a} t^{b}=\frac{1}{2} \delta^{a b}$. The pressure $p$ is given by the derivative of the partition function (3) with respect to the spatial volume, $V$,

$$
p=\frac{T}{V} \frac{\partial \log Z(T, V)}{\partial \log V}=-\frac{F}{V}=\frac{T}{V} \log \mathcal{Z}(T, V) .
$$

The last two equalities are valid for a sufficiently large and homogeneous system residing in thermodynamical equilibrium.

The energy density $\epsilon$ is given by the derivative of the partition function (3) with respect to the temperature $T$,

$$
\varepsilon=\frac{T}{V} \frac{\partial \log Z(T, V)}{\partial \log T} .
$$

The relation between the pressure (4) and the energy (5) constitutes the equation of state of the system, $p=p(\varepsilon)$.

According to Eqs. (4) and (5) it is sufficient to determine the partition function of the system in order to calculate the equation of state. However, available lattice simulations techniques are suitable for the calculation of quantum averages of operators rather than for the 
evaluation of the partition function itself. On the other hand, both the energy density $\varepsilon$ and the pressure $p$ can be derived from the quantum average of a single quantity, which is the trace of the energy-momentum tensor $T_{\mu \nu}$.

In $S U(N)$ gauge theory the energy-momentum tensor is given by the formula

$$
T_{\mu \nu}=2 \operatorname{Tr}\left[G_{\mu \sigma} G_{\nu \sigma}-\frac{1}{4} \delta_{\mu \nu} G_{\sigma \rho} G_{\sigma \rho}\right] .
$$

At the classical level the energy-momentum (6) is traceless because the bare Yang-Mills theory is a conformal theory. However, at the quantum level the conformal invariance is broken, and, consequently, the energymomentum tensor exhibits a trace anomaly: the quantum average of the trace of the energy-momentum tensor,

$$
\theta(T)=\left\langle T_{\mu}^{\mu}\right\rangle \equiv \varepsilon-3 p,
$$

is nonzero. The trace anomaly is intimately related to the gluon condensate which breaks the scale invariance of the theory

$$
\theta(T)=\left\langle\frac{\beta(g)}{2 g} G_{\mu \nu}^{a} G_{\mu \nu}^{a}\right\rangle,
$$

where the Gell-Mann-Low $\beta$-function is

$$
\beta(g)=\frac{\partial g}{\partial \log \mu}=-g^{3}\left(b_{0}+b_{1} g^{2}+\ldots\right),
$$

with one- and two-loop perturbative coefficients

$$
b_{0}=\frac{11 N}{3(4 \pi)^{2}}, \quad b_{1}=\frac{34 N^{2}}{3(4 \pi)^{4}} .
$$

The thermodynamic relations in Eqs. (4) and (5) relate the trace anomaly (77) with the pressure $p$,

$$
\theta(T)=T^{5} \frac{\partial}{\partial T} \frac{p(T)}{T^{4}}=-T^{5} \frac{\partial}{\partial T} \frac{\log \mathcal{Z}(T, V)}{T^{3} V} .
$$

Then, the pressure and, respectively, the energy density can be expressed via the trace anomaly, as follows:

$$
\begin{aligned}
& p(T)=T^{4} \int_{0}^{T} \frac{\mathrm{d} T_{1}}{T_{1}} \frac{\theta\left(T_{1}\right)}{T_{1}^{4}}, \\
& \varepsilon(T)=3 T^{4} \int_{0}^{T} \frac{\mathrm{d} T_{1}}{T_{1}} \frac{\theta\left(T_{1}\right)}{T_{1}^{4}}+\theta(T) .
\end{aligned}
$$

Equations (12) and (13) demonstrate that the trace anomaly (7) is the key quantity that allows us to reconstruct the whole equation of state.

The trace anomaly should vanish in a system of free massless relativistic particles

$$
\varepsilon^{\mathrm{SB}}=3 p^{\mathrm{SB}}=N_{\text {d.o.f. }} \frac{\pi^{2}}{30} T^{4},
$$

[where $N_{\text {d.o.f. }}=2\left(N^{2}-1\right)$ is the number of degrees of freedom in the noninteracting gas of the $S U(N)$ gluons] or in the case when excitations are too massive compared with the temperature, $m \gg T$ (then $\varepsilon \sim p \sim$ $\exp \{-m / T\}$ ). For Yang-Mills theory these statements imply that the dimensionless quantity $\theta / T^{4}$ should approach zero at very high temperatures (the gluons form a weakly interacting gas) and at very low temperatures (note that in $S U(N)$ Yang-Mills theories the mass gap is much greater than the critical temperature [27]). The latter property is used to fix the lower integration limits in Eqs. (12) and (13) at $T=0$.

\section{B. Lattice thermodynamics}

The lattice analogue of the partition function (3) of $S U(N)$ gauge theory is

$$
\mathcal{Z}(T, V)=\int D U \exp \left\{-\beta \sum_{P} S_{P}[U]\right\} .
$$

The plaquette action $S_{P}[U]$ of the gluonic link fields $U_{x \mu}$ is usually written in the Wilson form,

$$
S_{P}[U]=1-\frac{1}{N} \operatorname{Re} \operatorname{Tr} U_{P}
$$

The identification of the lattice results with the physics in continuum is achieved in the limit of vanishing lattice spacing, $a \rightarrow 0$ :

$$
\begin{aligned}
U_{x \mu} & =\exp \left\{i g \int_{x}^{x+a \hat{\mu}} \mathrm{d} y A_{\mu}(y)\right\} \\
& \rightarrow \mathbb{1}+i a g A_{\mu}(x)+O\left(a^{2}\right),
\end{aligned}
$$

The lattice spacing $a$ is a function of the lattice coupling

$$
\beta=2 N / g^{2} \text {. }
$$

The spatial volume $V=\left(L_{s} a\right)^{3}$ and the temperature

$$
T=\frac{1}{L_{t} a}
$$

of the gluonic system are related to the asymmetric geometry of the Euclidean lattice, $L_{s}^{3} L_{t}$. The shorter direction, $L_{t}$ with $L_{t} \leqslant L_{s}$, is associated with the imaginary time, or, "temperature" direction. The imaginary time formalism allows us to calculate various thermodynamic quantities corresponding to the gauge system residing in thermodynamic equilibrium at given temperature and volume. Using the relation $T(\partial / \partial T)=-a(\partial / \partial a)$ one can rewrite the continuum expression for the anomaly (11) in a form suitable for numerical lattice simulations,

$$
\frac{\theta(T)}{T^{4}}=6 L_{t}^{4}\left(\frac{\partial \beta(a)}{\partial \log a}\right) \cdot\left(\left\langle S_{P}\right\rangle_{T}-\left\langle S_{P}\right\rangle_{0}\right)
$$

Here the plaquette averages $\left\langle S_{P}\right\rangle_{T}$ and $\left\langle S_{P}\right\rangle_{0}$ are the action densities taken, respectively, in a thermal bath at 
$T>0\left(L_{s}^{3} L_{t}\right.$ lattices $)$ and at $T=0$ ( $L_{s}^{4}$ lattices $)$. In Eq. (20) it is implied that the $T=0$ plaquette expectation value is subtracted to remove the effect of quantum fluctuations, which lead to an ultraviolet (UV) divergency of the quantum expectation value. As a result, the trace anomaly becomes a UV-finite quantity, which is normalized to zero at $T=0$ because of the existence of the mass gap. Equation (20) is a lattice version of Eq. (8). The trace anomalies and the equation of state for $S U(2)$ and $S U(3)$ gauge theories were calculated in Refs. 24] and [25], respectively.

The anomaly (20) can be separated into electric and magnetic parts, respectively,

$$
\theta(T)=\theta_{E}(T)+\theta_{M}(T) .
$$

The electric contribution comes from the field strength tensors of the chromoelectric fields, $E_{i}^{a}=G_{i 4}^{a}$, while the magnetic part is solely due to chromomagnetic fields, $B_{i}^{a}=(1 / 2) \epsilon_{j k} G_{i j k}^{a}$. On the lattice the former is associated with temporal plaquettes, $P_{t} \equiv P_{i 4}$ with $i=1,2,3$, while the latter is related to the spatial plaquettes, $P_{s} \equiv$ $P_{i j}$ with $i<j=1,2,3$ :

$$
\begin{aligned}
& \frac{\theta_{E}(T)}{T^{4}}=3 L_{t}^{4}\left(\frac{\partial \beta(a)}{\partial \log a}\right) \cdot\left(\left\langle S_{P_{t}}\right\rangle_{T}-\left\langle S_{P}\right\rangle_{0}\right), \\
& \frac{\theta_{M}(T)}{T^{4}}=3 L_{t}^{4}\left(\frac{\partial \beta(a)}{\partial \log a}\right) \cdot\left(\left\langle S_{P_{s}}\right\rangle_{T}-\left\langle S_{P}\right\rangle_{0}\right) .
\end{aligned}
$$

Summarizing, the only lattice observable which is needed for the calculation of the trace anomaly (20) [including its electric (22) and magnetic (22) parts] and, consequently, for the determination of thermodynamics of Yang-Mills theory, is the difference between the expectation values of the action densities calculated at zerotemperature (at $L_{t}=L_{s}$ ) and at finite temperature (at $L_{t}<L_{s}$ ). The $\beta$-function in Eq. (201) has to be determined from independent lattice simulations.

\section{SU(2) THERMODYNAMICS AT MODERATE LATTICES}

In this Section we present basic results for the thermodynamics of $S U(2)$ gauge fields. The thermodynamics of $S U(2)$ lattice Yang-Mills theory has been studied in detail in Ref. 24]. In this Section we repeat certain numerical calculations of Ref. [24] at moderate sized lattices which will be used in the subsequent Section in the investigation of the vortex contributions to the thermodynamics of the system.

We are working on the lattices $18^{3} \times 4$ and $18^{4}$ corresponding to finite- and zero-temperature cases, respectively. At the asymmetric lattice with $L_{t}=4$ the thermal phase transition is realized at $\beta=\beta_{c} \approx 2.3$, Ref. [26]. In physical units the transition in $S U(2)$ gauge theory is achieved at temperature [27] $T_{c}=305(8) \mathrm{MeV}$, if one fixes the scale by setting the $T=0$ tension of the chromoelectric string at the phenomenologically accepted value of $\sigma^{1 / 2}=440 \mathrm{MeV}$.

\begin{tabular}{|c|c|c|c|c|c|c|c|}
\hline$\beta$ & 2.27 & 2.3 & 2.35 & 2.4 & 2.45 & 2.53 & 2.66 \\
\hline$T / T_{c}$ & 0.909 & 1.005 & 1.188 & 1.401 & 1.651 & 2.14 & 3.24 \\
\hline$a, \mathrm{fm}$ & 0.183 & 0.165 & 0.140 & 0.119 & 0.101 & 0.0777 & 0.0513 \\
\hline$L_{s} a, \mathrm{fm}$ & 3.293 & 2.977 & 2.520 & 2.136 & 1.813 & 1.399 & 0.9234 \\
\hline$-\frac{\partial \beta}{\partial \log a}$ & 0.2965 & 0.2982 & 0.3011 & 0.3038 & 0.3064 & 0.3103 & 0.3160 \\
\hline
\end{tabular}

TABLE I: Parameters of our numerical simulations: temperature (in units of the critical temperature $T_{c}$ ), lattice spacing $a$, and spatial extension $L_{s} a$ for selected values of the lattice coupling $\beta$.

The gauge field configurations are generated using the standard Wilson action (16). In order to determine the lattice spacing $a$ as a function of the lattice gauge coupling $\beta$ we use the interpolating function of Ref. [28] which describes with a high accuracy various lattice calculations of $a$. The lattice spacing $a$ in units of the $T=0$ string tension $\sigma$ is parameterized as follows:

$$
\ln \left(a^{2} \sigma\right)=-\frac{64 \pi^{4} b_{0}}{\beta}+\frac{b_{1}}{b_{0}^{2}} \ln \frac{64 \pi^{4} b_{0}}{\beta}+\frac{d}{3 b_{0} \beta}+c .
$$

The first two terms in the right hand side (r.h.s.) of the above equations represent, respectively, the first and the second order loop corrections coming from the perturbation theory. The coefficients $b_{0}$ and $b_{1}$ are the coefficients of the $\beta$-function (9) which are given by Eq. (10) with $N=2$. The other two terms in the r.h.s. of Eq. (24) are introduced to mimic higher-order perturbative corrections as well as nonperturbative effects. It was found 28] that the choice $c=4.38(9)$ and $d=1.66(4)$ reproduces measured valued of the lattice spacing $a$ very well.

We use the interpolating relation (24) in order to determine the temperature (19) and the prefactor in the r.h.s. of Eq. (20). In Table \we present several important quantities inherent to our numerical calculations.

In Fig. 2 we show the trace anomaly (20) along with its electric (22) and magnetic (23) parts. It is very interesting to notice that the magnetic contribution to the anomaly vanishes in the deconfinement phase at a temperature approximately twice larger than the transition temperature,

$$
\theta_{M}\left(T_{0}\right)=0, \quad T_{0}=2.15(1) T_{c} .
$$

This distinguished value of the temperature is also seen from the asymmetry of the so-called $A^{2}$-condensate: the thermal fluctuations of the electric and magnetic components of the gluonic fields in the Landau gauge becomes equal around $2 T_{c}$, see Ref. 29] for details. In the case of the $S U(3)$ gauge group the magnetic contribution to the trace anomaly also vanishes around the temperature which is slightly higher than $2 T_{c}$, Ref. [25]. The mentioned coincidence allows us to conjecture that the value (25) of the critical temperature, $T \approx 2 T_{c}$, is in fact universal for all numbers of colors $N$. 


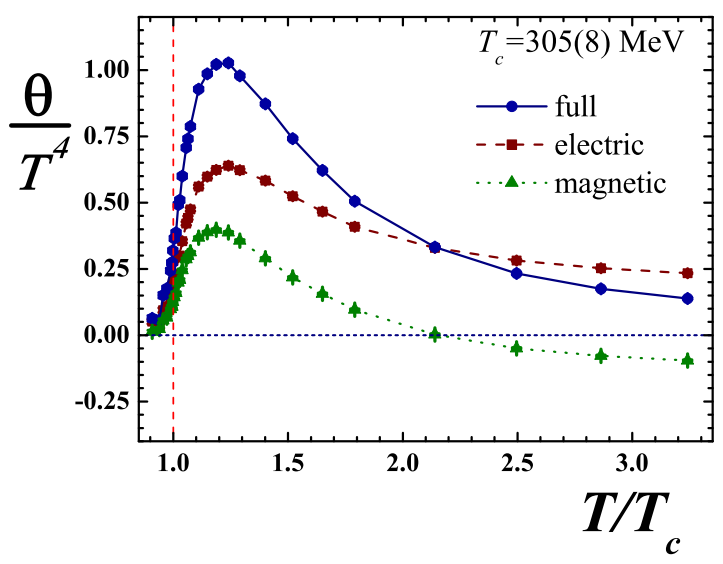

FIG. 2: (Color online) The trace anomaly $\theta$, represented as a dimensionless ratio $\theta / T^{4}$, as a function of the temperature $T$ (in units of the critical temperature, $T_{c}$ ). We show the full anomaly (circles), and its electric (squares) and magnetic (triangles) contributions determined by Eqs. (20), (22) and (23), respectively.

\section{THERMODYNAMICS OF VORTICES}

\section{A. Magnetic vortices and gluon trace anomaly: lattice definitions}

As we have discussed in the Introduction, (magnetic) center vortices are stringlike configurations of the gauge fields which are relevant for color confinement in the lowtemperature confinement phase. In this Section we investigate how these objects contribute to the thermodynamics of the $S U(2)$ gauge system.

The magnetic vortices carry a magnetic flux characterized by a nontrivial center element of the gauge group. Such objects can conveniently be found in a specific gauge, which makes the gauge field as close to the center subgroup of the gauge group as possible. In $S U(2)$ Yang-Mills theory the relevant gauge - often called as the (direct) maximal center gauge - is defined by a maximization of the functional

$$
\max _{\Omega} F\left[U^{\Omega}\right], \quad F[U]=\sum_{l}\left(\operatorname{Tr} U_{l}\right)^{2},
$$

with respect to the gauge transformations

$$
U_{x \mu} \rightarrow U_{x \mu}^{\Omega}=\Omega_{x} U_{x \mu} \Omega_{x+\hat{\mu}}^{\dagger} .
$$

In Eq. (26) the sum goes over all links $l$ of the lattice.

The center element of the gauge field $U_{l}$ can be identified after the $S U(2)$ maximal center gauge (26) is fixed:

$$
Z_{l}=\operatorname{sign} \operatorname{Tr} U_{l}= \pm 1, \quad Z_{l} \in \mathbb{Z}_{2}
$$

The maximal center condition (26) fixes the gauge freedom (27) up to a center subgroup of the $S U(2)$ gauge group:

$$
U_{x \mu} \rightarrow U_{x \mu}^{\omega}=\omega_{x} U_{x \mu} \omega_{x+\hat{\mu}}, \quad \omega_{x} \in \mathbb{Z}_{2} .
$$

In terms of the $\mathbb{Z}_{2}$ gauge variables (28) the center transformation (29),

$$
Z_{x \mu} \rightarrow Z_{x \mu}^{\omega}=\omega_{x} Z_{x \mu} \omega_{x+\hat{\mu}}
$$

can naturally be interpreted as a $\mathbb{Z}_{2}$ transformation in a $\mathbb{Z}_{2}$ effective gauge theory written in terms of the gauge fields (28).

The field strength tensor of the center gauge fields (28) is the $\mathbb{Z}_{2}$ plaquette

$$
Z_{P}=Z_{1} Z_{2} Z_{3} Z_{4}, \quad Z_{P} \in \mathbb{Z}_{2}
$$

where the subscripts $1 \ldots 4$ denote the links forming the border of the plaquette $P$. The $\mathbb{Z}_{2}$-field strength tensor (31) is invariant under the $\mathbb{Z}_{2}$ gauge transformations 30 .

The vortex worldsheets $\Sigma$ are identified with the help of the $\mathbb{Z}_{2}$-plaquette (31). Let us introduce the notation ${ }^{*} P$ for the plaquette which belongs to the dual lattice and which is dual to the plaquette $P$. Then the dual plaquette ${ }^{*} P$ does not contain the center vortex if the corresponding plaquette is center-trivial, $Z_{P}=+1$. Equivalently, one may say that no vortex is going through the plaquette ${ }^{*} P$, if $Z_{P}=+1$. However, if $Z_{P}=-1$, the plaquette ${ }^{*} P$ is a part of the vortex world-sheet. In short,

$$
Z_{P}= \begin{cases}-1, & \text { if } P \in \Sigma \\ +1, & \text { if } P \notin \Sigma\end{cases}
$$

One can easily prove that vortices are closed loops since their worldsheets are closed surfaces.

Using Eq.(32) one can define the lattice vortex density

$$
\rho_{P}=\frac{1}{2}\left(1-Z_{P}\right)= \begin{cases}1, & \text { if } P \in \Sigma \\ 0, & \text { if } P \notin \Sigma\end{cases}
$$

Then the averaged lattice vortex density is

$$
\rho=\frac{1}{2}\left(1-\left\langle Z_{P}\right\rangle\right) .
$$

The lattice fields can be decomposed into the vortex singular contribution and the rest

$$
U_{l}=Z_{l} \tilde{U}_{l},
$$

where the $S U(2)$ gauge field $\tilde{U}_{l}$ is defined as follows

$$
\operatorname{Tr} \tilde{U}_{l}>0 \text {. }
$$

The trace of the $S U(2)$ plaquette (which is an explicitly $S U(2)$ invariant quantity), can be decomposed into a singular (center-valued) contribution and a regular contribution, respectively,

$$
\operatorname{Tr} U_{P}=Z_{P} \operatorname{Tr} \tilde{U}_{P}, \quad \tilde{U}_{P}=\tilde{U}_{1} \tilde{U}_{2} \tilde{U}_{3}^{\dagger} \tilde{U}_{4}^{\dagger}
$$

This relation is very useful for us since it allows us to identify the contribution of the singular magnetic vortices into the vacuum expectation value (vev) of the 
$S U(2)$ plaquette $\left\langle\operatorname{Tr} U_{P}\right\rangle$. This quantity is related to the vev of the gluonic action (16), which, in turn, contributes to the thermodynamics of the system via the trace anomaly (20).

In order to calculate the contribution of magnetic vortices into the trace anomaly (20), and, consequently, into the equation of state, we utilize the following chain of considerations. For any given configuration of the gauge field $U_{l}$ one can identify the location of the center vortex worldsheet, ${ }^{*} \Sigma$, as we described above. The worldsheet ${ }^{*} \Sigma$ is a collection of plaquettes ${ }^{*} P$ of the dual lattice, which forms a closed surface, Figure 3. Then the set of all

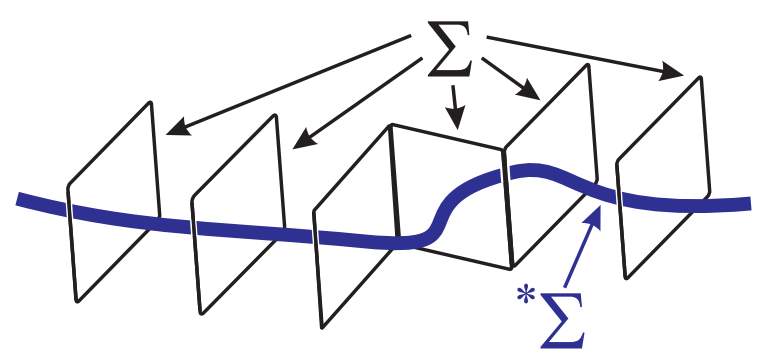

FIG. 3: (Color online) A three dimensional visualization of the magnetic vortex string ${ }^{*} \Sigma$ which pierces a set of the plaquettes $P \in \Sigma$.

plaquettes of the lattice can be separated into two (generally, unequal) subsets: (i) the plaquettes $P$ of the original lattice belonging to the vortex worldsheet, $P \in \Sigma$; and (ii) the plaquettes $P$ which are outside of vortices, $P \notin \Sigma$. The total action of the gauge field configuration can equivalently be decomposed into the sum of the contributions of the individual plaquettes belonging to the vortex worldsheet and the sum going over plaquettes outside of vortices, respectively:

$$
\begin{aligned}
\sum_{P} S_{P} & =\sum_{P \in \Sigma} S_{P}+\sum_{P \notin \Sigma} S_{P} \\
& \equiv \sum_{P} \rho_{P} S_{P}+\sum_{P}\left(1-\rho_{P}\right) S_{P}
\end{aligned}
$$

Then we divide both sides of Eq. (39) by the total number of the lattice plaquettes, $6 L_{s}^{3} L_{t}$, and average over all gauge field configurations. As a result, we get that the average gluonic action per plaquette is given by the sum

$$
\left\langle S_{P}\right\rangle=\left\langle S_{P}\right\rangle^{\text {vort }}+\left\langle S_{P}\right\rangle^{\text {novort }},
$$

where $\left\langle S_{P}\right\rangle^{\text {vort }}$ is the vev of the gauge action coming from the vortex worldsheets,

$$
\begin{aligned}
\left\langle S_{P}\right\rangle^{\text {vort }} & =\left\langle\rho_{P} S_{P}\right\rangle \equiv \frac{1}{6 L_{s}^{3} L_{t}}\left\langle\sum_{P} \rho_{P} S_{P}\right\rangle \\
& =\rho+\frac{1}{4}\left(\left\langle\operatorname{Tr} \widetilde{U}_{P}\right\rangle-\left\langle\operatorname{Tr} U_{P}\right\rangle\right),
\end{aligned}
$$

and the action coming from the space unoccupied by the vortex worldsheets,

$$
\begin{aligned}
\left\langle S_{P}\right\rangle^{\text {novort }} & =\left\langle\left(1-\rho_{P}\right) S_{P}\right\rangle \\
& \equiv \frac{1}{6 L_{s}^{3} L_{t}}\left\langle\sum_{P}\left(1-\rho_{P}\right) S_{P}\right\rangle \\
& =1-\rho-\frac{1}{4}\left(\left\langle\operatorname{Tr} \widetilde{U}_{P}\right\rangle+\left\langle\operatorname{Tr} U_{P}\right\rangle\right) .
\end{aligned}
$$

In order to derive the relations Eq. (41) and (42) we used Eqs. (33), (34) as well as Eq. (16) for the case of two colors, $N=2$. Then we utilized the relations,

$$
\begin{aligned}
\rho_{P} S_{P} & =\rho_{P}+\frac{1}{4} Z_{P} \operatorname{Tr} U_{P}-\frac{1}{4} \operatorname{Tr} U_{P}, \\
\left(1-\rho_{P}\right) S_{P} & =\left(1-\rho_{P}\right)-\frac{1}{4} Z_{P} \operatorname{Tr} U_{P}-\frac{1}{4} \operatorname{Tr} U_{P},
\end{aligned}
$$

and used the fact that, according to Eq. (37),

$$
Z_{P} \operatorname{Tr} U_{P}=\operatorname{Tr} \widetilde{U}_{P}
$$

It is important to realize that the vev of the quantity (45) can be interpreted as the vev of the plaquette $\operatorname{Tr} U_{P}$ evaluated on configurations with "removed vortices". Indeed, if we manually set $Z_{P}=1$ at all plaquettes of the gauge field configuration, then the vacuum expectation of $\operatorname{Tr} U_{P}$ at these modified configurations is automatically equivalent to the vev of $\operatorname{Tr} \widetilde{U}_{P}$ at the original (unmodified) configuration:

$$
\left\langle\operatorname{Tr} \widetilde{U}_{P}\right\rangle \equiv\left\langle Z_{P} \operatorname{Tr} U_{P}\right\rangle \equiv\left\langle\operatorname{Tr} U_{P}\right\rangle_{\text {removed vortices }}
$$

The procedure of "removing vortices" is equivalent to a shift of the gluonic link fields $U_{l}$,

$$
U_{l} \rightarrow Z_{l} U_{l} \equiv \tilde{U}_{l}, \quad Z_{l}=\operatorname{sign} \operatorname{Tr} U_{l},
$$

according to the decomposition (35). By definition, the thus-modified configuration contains no center vortices. Note that the gauge field configurations with removed vortices are not able to support the color confinement [30].

Summarizing, the lattice density of the gluonic action - which is crucial for the determination of the trace anomaly (20) - can be split according to Eq. (40) into a piece coming from the vortex worldsheets (41) and arising from the regions of space lying outside of vortices (42). In the next Subsections we analyze these contributions in more details.

\section{B. Center vortices and action density: numerical results}

We have studied vortex properties on the lattices $18^{4}$ and $18^{3} \times 4$. Depending on the value of the lattice coupling $\beta$ we used from 100 to 500 statistically independent configurations for the zero temperature lattice $18^{4}$, and from 700 to 1600 configuration for the finite-temperature 
lattice $18^{3} \times 4$. Generally, the larger $\beta$ the larger is the number of gauge field configurations needed to reach an acceptable statistical accuracy of the numerical results. The maximal center gauge (26) was fixed using a simple iterative algorithm. In our simulations we considered one Gribov copy only. Despite the unsophisticated nature of our approach, we believe - following the results reported in Ref. [31] and in Ref. 32 - that this algorithm allows us to capture not only qualitative but also, to a large extent, various quantitative features of the vortex ensembles at finite temperature. In our visualization of the vortex-related data we indicate the statistical errors only, leaving aside the (systematical) error bars related to the gauge fixing issues such as the choice of the Gribov copy.

Below we present results of our numerical simulations for several important quantities calculated both in lattice and in physical units. Before proceeding to the calculation of the trace anomaly and other related quantities, we discuss the important numerical results for the density of the magnetic (center) vortices.

\section{Vortex density}

At finite temperature the vortex density $\rho$ was studied in Ref. [19, 20, 21, 22]. We calculate the vortex density both at finite temperature and at zero-temperature in order to disentangle the finite-temperature effects from the effects related to the variation of the UV cutoff. Indeed, the UV cutoff - the role of which is played by the (inverse) lattice spacing $a$ - is controlled by the lattice coupling constant $\beta$, as, for example, illustrated by the interpolating function (24). The temperature is related to the extension of the lattice in the short direction (19). The comparison of the lattice results obtained at different lattice geometries and at the same fixed value of $\beta$ allows us to single out effects of temperature variations on the vortex density.

In Figure 4 we show the total density of the center vortices (34) calculated on the lattices $18^{4}$ and $18^{3} \times 4$. One can observe that in the confinement phase, $\beta<\beta_{c} \approx$ 2.3 , the vortex density is almost the same for $L_{t}=18$ and $L_{t}=4$ lattices.

In the deconfinement phase, $\beta>\beta_{c}$ on the $L_{t}=4$ lattice, the vortex density starts to deviate from its zerotemperature value (calculated on the $L_{t}=18$ lattice). It is quite surprising to notice that just above the phase transition the density of the thermal vortices gets lower compared with the value of the vortex density at zerotemperature. However, as the temperature (or, equivalently, the value of the lattice gauge coupling $\beta$ on the $L_{t}=4$ lattice) increases further, the absolute value of the difference in the vortex densities at $L_{t}=4$ and $L_{t}=18$ lattices reaches its maximum, and then the deviation between the vortex densities starts to decrease again. Thus, the effect of the temperature on the vortex density is quite nontrivial.

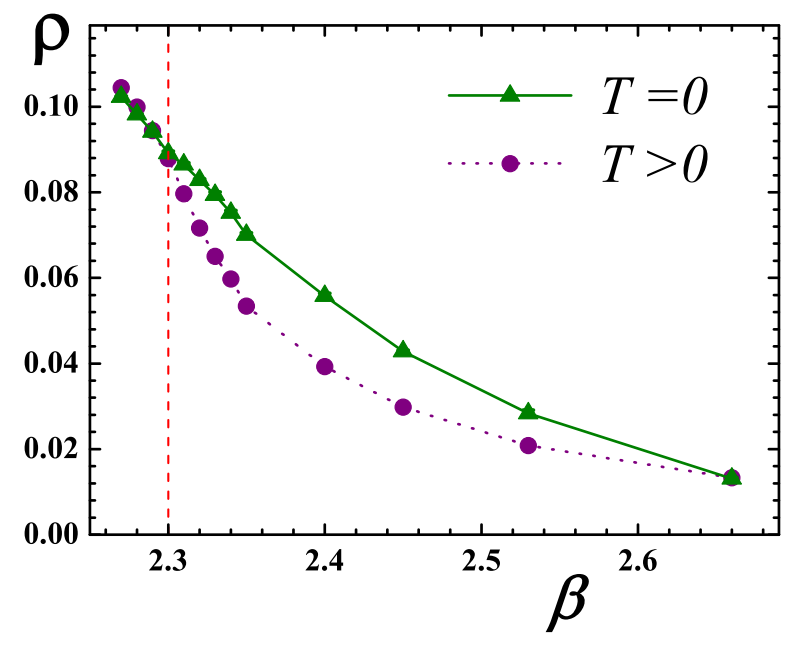

FIG. 4: (Color online) The lattice density of the center vortices (34) at zero-temperature (the solid line) and at finite temperature (dotted line) vs the gauge coupling $\beta$. The simulations are done on $18^{4}$ and $18^{3} \times 4$ lattices, respectively. The vertical dashed line marks the critical coupling $\beta_{c}$ corresponding to the finite-temperature phase transition. On the $18^{3} \times 4$ lattice, a lower value of $\beta$ corresponds to a lower temperature. Hereafter some sets of the numerical data points are connected by lines to guide the eye.

At zero-temperature the vortex ensembles typically consist of one very long vortex worldsheet which spreads over almost the whole spacetime, and a lot of short-sized clusters of vortex worldsheets. The worldsheet of the infinitely long vortex trajectory is usually associated with a condensed (infrared) vortex component while the short UV clusters form a perturbative (UV) component. It is the condensate of vortices which leads to the confinement of color charges. At $T>T_{c}$ the spatial string tension in the deconfinement phase is supported by the vortex percolation in the spatial dimensions $[19,21]$.

At finite temperature it looks natural to separate the strings in thermodynamic ensembles into two components describing, respectively, the virtual strings and the real strings. It is easy to explain the meaning of "virtual" and "real" in terms of particlelike objects which we understand much better than vortices which are stringlike defects [6]: the real objects are thermodynamically relevant while the virtual ones are not. In other words, the virtual objects are associated with the ground state of the theory realized at zero-temperature while the real particles are the excitations of this ground state.

Consider, for example, the Abelian monopoles at finite temperature in Euclidean Yang-Mills theory. At zerotemperature a part of the monopoles is condensed (similarly to vortices) while another part of the monopole trajectories belong to ultraviolet clusters. At finite temperature the ensembles of the monopole trajectories should contain both the virtual monopoles belonging to the vac- 
uum and, at the same time, the thermodynamically relevant (or, real) monopoles. In order to measure physically meaningful observables in the continuum limit, one should be able to separate these types of monopoles from each other. According to Ref. [6] the thermodynamically relevant monopoles can be distinguished from the virtual monopoles by a simple principle: the thermal monopoles should wrap around the temperature (compactified) direction of the Euclidean space. Moreover, the quantum density of the thermal monopoles is not equal to the average density of the total length of the (wrapped) monopole trajectories as one could naïvely guess on general grounds. Such a quantity is divergent in the UV regime making its interpretation somewhat obscure. The density of the thermal monopoles corresponds to the density of the winding number $s$ of the monopole trajectories in the temperature direction of the Euclidean spacetime:

$$
\rho_{\text {mon }}^{\text {thermal }}=\frac{1}{V_{3 d}}\langle|s|\rangle .
$$

Coming back to the magnetic vortices, one can ask the important question: how to separate the thermal component of the vortex density from the density of the virtual component? In other words: in Euclidean lattice simulations at finite temperature we observe a set of closed vortex trajectories in each configuration of the gauge fields. Which vortex worldsheets are real (thermal) and which worldsheets corresponds just to virtual strings? Following our experience with particlelike monopoles [ [] one can suggest that the worldsheets of the real vortices are characterized by a nontrivial wrapping number with respect to the (compactified) temperature dimensions.

A vortex analogue of Eq. (48) remains to be derived. Note that the naive suggestion for the thermal vortex density - the averaged area of the wrapped vortex worldsheets per unit Euclidean volume - in general should not be correct. In fact, in Ref. [6] it was shown that the analogous quantity for the monopoles (the average length of wrapped monopole trajectories per unit Euclidean volume) is - contrary to the correct expression (48) - divergent in the UV limit and thus cannot serve as the density of the real monopoles.

Let us stress again a somewhat puzzling behavior of the vortex density, Figure 4. Naively, in a quantum field theory an expectation value of a local quantity at finite temperature should receive contributions from both quantum field fluctuations - which are inherent solely to the zerotemperature ground state - and from various thermal excitations. The (virtual) quantum field fluctuations have the same structure both at zero and at finite temperature (for example, the UV divergencies both at zero and at finite temperatures are identical [33]). Therefore, in order to figure out a contribution of the thermal excitations one can fondly subtract a zero-temperature vev from the thermal expectation value. This subtraction, for example, is routinely performed for the trace anomaly of the Yang-Mills fields (20). The puzzle is that if the subtraction procedure is applied to the vortex density, then the would-be thermal vortex density takes a negative value in the deconfinement phase, Figure 5. The negative valued quantity is difficult to interpret in terms of the density of realistic objects. Thus, the density of the thermal (real) vortices needs to be defined using a nonstandard way like it is done for the particlelike objects (48) in Ref. [6]. Note, that in our simulations we do not discriminate the thermal (wrapped) and the UV vortex components.

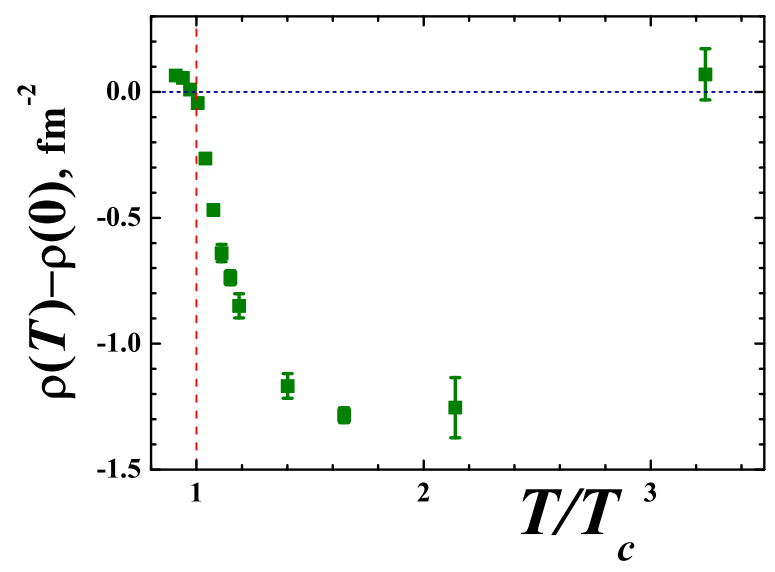

FIG. 5: (Color online) The difference between the vortex density at finite temperature and at zero-temperature (physical units).

One can observe a few interesting features of the difference in the vortex densities shown in Figure 5 .

- The difference in the vortex densities is positive in the confinement phase, $T<T_{c}$.

- This quantity is negative in the deconfinement phase up to temperatures of the order of, approximately, three critical temperatures, $T_{c}<T \lesssim 3 T_{c}$. At higher temperatures the quantity will again take positive values.

- The minimum of the thermal density is reached at $T \approx 2 T_{c}$. Note that this is the temperature at which the contribution of the magnetic gluons vanishes, see Eq. (25) and Figure 2.

\section{Comment on monopoles vs vortices}

The fact that the minimum of the vortex density is reached at $T \approx 2 T_{c}$ is intriguing. As we have discussed above, magnetic vortices are related to magnetic monopoles. According to Ref. [6] the condensed (in the confinement phase) state of the monopoles melts into a monopole liquid at $T=T_{c}$ which is evaporated into a monopole gas at $T \approx 2 T_{c}$, at which the vortex density reaches its minimum. The coincidence of the temperatures may be not accidental. An illustration of the monopole states in the finite-temperature Yang-Mills theory is provided in Figure 6 . 


\begin{tabular}{|l|c|ccc|}
\hline condensate & liquid & \multicolumn{3}{|c|}{ gas } \\
\hline 0 & 1 & 2 & 3 & $T / T_{c}$
\end{tabular}

FIG. 6: (Color online) The states of the monopole matter in Yang-Mills theory at finite temperature according to Ref. [6] .

The existence of the monopole liquid close to the critical point in the deconfinement phase was confirmed by accurate lattice data of Ref. [34] according to an analysis presented in Ref. [35]. The appearance of the monopole gas at even higher temperatures - discussed earlier in analytical terms in Ref. 8] - was demonstrated numerically in Ref. [36]. We refer the interested reader to Ref. [17] for a short review of this subject.

At very high temperatures the general behavior of the vortex density can be predicted from dimensional reduction arguments. According to the dimensional reduction, at high temperatures nonperturbative physics is controlled by three-dimensional magnetodynamics which corresponds to zero Matsubara frequency of the original four-dimensional theory. The vortex trajectories become static, and the vortex density becomes equal to the density of the vortex lines in the $3 d$ space. The magnetodynamical quantities are expressed in terms of the corresponding powers of the gauge coupling in the dimensionally reduced theory,

$$
g_{3 d}^{2}(T)=T g_{4 d}^{2}(T)
$$

where $g_{4 d}$ is the running coupling of the original fourdimensional theory calculated at the scale $T$.

Thus, density of vortices in the high- $T$ limit should be described by the formula,

$$
\rho(T)=C_{\mathrm{vort}} g_{3 d}^{4}(T) \propto\left(\frac{T}{\log T / \Lambda}\right)^{2}, \quad T \gg T_{c}
$$

where $C_{\text {vort }}$ is a temperature-independent parameter and $\Lambda \sim \Lambda_{\mathrm{QCD}}$ is a dimensional parameter.

The power, with which the temperature enters Eq. (50), is clear from the dimensional arguments, $\rho \sim O\left(T^{2}\right)$. However, the logarithmic behavior, $\log ^{-\alpha_{\text {vort }}}(T / \Lambda)$, with $\alpha_{\text {vort }}=2$ is governed by the perturbation theory. It is interesting to note that the same power of the logarithm, $\alpha \approx 2$, was recently found for the Abelian monopoles in the high-temperature phase of the Yang-Mills theory [34]. This fact is amusing, because for the monopoles, which are particlelike objects, the natural power of the logarithm would be $\alpha_{\text {mon }}=3$, Ref. [6, 88]. The observed difference allowed the authors of Ref. 34] to conclude that the monopoles do not form a free particle gas. We support this opinion by noticing, that the monopoles are to be related to the magnetic vortices, and the vortex dynamics make the monopole properties less trivial. This could explain why the observed power of the monopole logarithm, coincides with the vortex one,

$$
\alpha_{\text {mon }} \approx \alpha_{\text {vort }}=2 .
$$

\section{Expectation value of $S U(2)$ plaquettes}

We have calculated numerically the expectation values of the $S U(2)$ plaquettes $\frac{1}{2} \operatorname{Tr} U_{P}$ at the original configurations and compared them with the vortex-removed configurations. The comparison was done both at zero and finite temperature. The plaquette expectation values are crucial for the determination of contribution of vortices into the expectation value of the gluon action, Eqs. (41), (42), and (46). Notice, that according to Eq. (16), the larger the plaquette expectation value the smaller the action density (and vice versa).

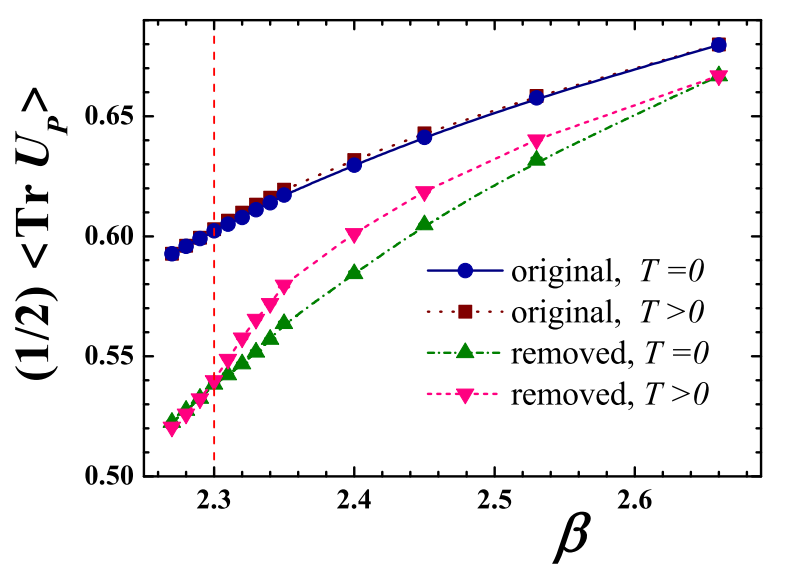

FIG. 7: (Color online) The expectation values of the plaquette $\frac{1}{2} \operatorname{Tr} U_{P}$ at zero and at finite temperatures for usual and for vortex-removed configurations.

The expectation value of the $S U(2)$ plaquettes is presented in Figure 7 One can immediately observe the following interesting features:

- The plaquette expectation values for configuration with removed vortices is much smaller compared to the original unmodified configurations. This feature is valid both at zero and at finite temperatures in all phases.

- Thus, magnetic vortices carry positively valued energy density. This fact was already established for $T=0$ Yang-Mills theory in Ref. [37].

- The temperature fluctuations increase the plaquette expectation value both at the original configurations and at the modified gauge field configurations.

- Thus, the effect of the temperature is to decrease the energy density both at the vortex worldsheets and at the space outside of vortices.

- The influence of the temperature on the plaquette expectation values of the vortex-removed configurations is 
much stronger compared with the observed influence of the temperature on the original gauge field configurations.

- Thus, the energy densities at the vortex worldsheets and outside of vortices receive very strong contributions due to thermal configurations. These contributions are canceled in the original configurations while they cannot be canceled in the modified configurations due to the removal of the vortices.

This observation stresses the significance of the vortices for the thermodynamics of the gauge system.

Using the numerical data for the vortex density, for the plaquette expectation values both at the original configurations and at the vortex-removed configurations one can calculate the contribution of the vortices to the vacuum expectation value of the gluon action density. The contribution of the vortices - calculated with the help of Eq. (41) - is presented in Figure 8, It is clearly seen that the effect of the temperature is to decrease the total action carried by the vortices.

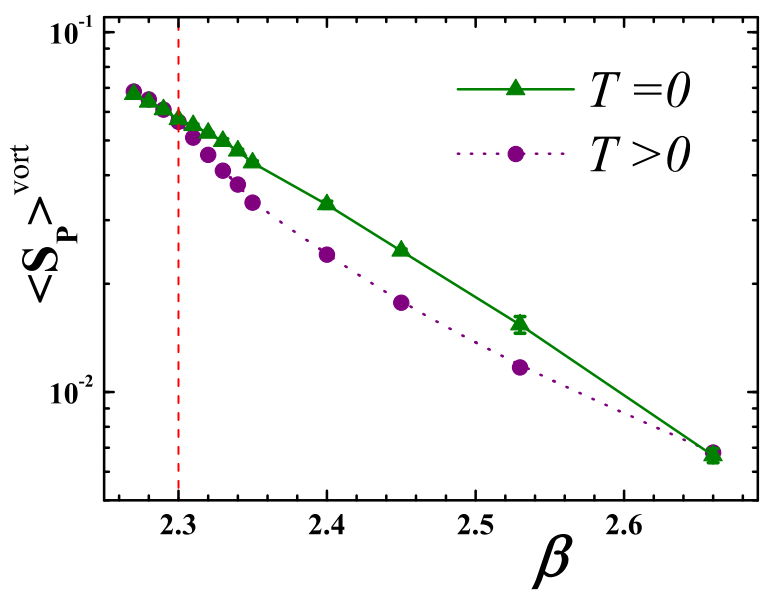

FIG. 8: (Color online) The expectation value of the action density calculated at the vortex worldsheets (41) at zero temperature $\left(18^{4}\right.$ lattice, the solid line $)$ and at finite temperature $\left(18^{3} \times 4\right.$ lattice, the dotted line) vs the gauge coupling $\beta$.

It is very important to stress, that we have observed two different temperature effects which are realized just above the critical temperature in the deconfinement phase $\left(T_{c}<T \lesssim 2 T_{c}\right)$ :

- According to Figure 8 the higher the temperature the lower the contribution of the vortices to the vev of the action density.

- According to Figure 4 the higher the temperature the lower the vortex density.

The knowledge of these facts alone does not allow us to conclude what is the primary reason of the diminishing of the vortex contribution into the total action density. For example, the energy density per unit area of the vortex worldsheet could be insensitive to the temperature and the overall effect could be due to the lowering of the vortex density with increase of temperature. Other options are: the energy density at the worldsheet may moderately increase (or, decrease) with temperature weakening (enhancing) the effect of the dropping vortex density. The same question may be addressed at higher temperatures, $T \gtrsim 2 T_{c}$, at which both the vortex density and the thermal plaquette values are increasing functions of temperature.

We study numerically the action density per an elementary plaquette belonging to the vortex worldsheet ${ }^{1}$ :

$$
\left\langle s_{P}\right\rangle^{\mathrm{vort}}=\frac{\left\langle S_{P}\right\rangle^{\mathrm{vort}}}{\rho} \equiv \frac{\left\langle\sum_{P} \rho_{P} S_{P}\right\rangle}{\left\langle\sum_{P} \rho_{P}\right\rangle},
$$

the action density per an elementary plaquette outside of the vortex worldsheet

$$
\left\langle s_{P}\right\rangle^{\text {novort }}=\frac{\left\langle S_{P}\right\rangle^{\text {novort }}}{1-\rho} \equiv \frac{\left\langle\sum_{P}\left(1-\rho_{P}\right) S_{P}\right\rangle}{\left\langle\sum_{P}\left(1-\rho_{P}\right)\right\rangle} .
$$

For the sake of brevity we call the quantity (52) as "the specific vortex action" because it represents the gluonic action density normalized by the area of the vortex worldsheet. Analogously, we refer to the quantity (52) as to "the specific no-vortex action".

The results - which are represented in Figure 9 - are quite interesting:

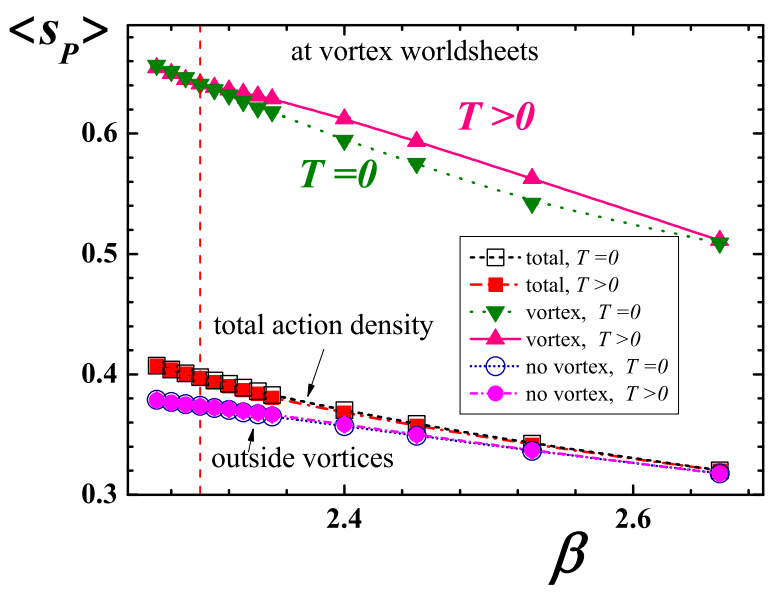

FIG. 9: (Color online) The same as in Figure 8 but for the action density per an elementary plaquette belonging to the vortex worldsheet (52) and per an elementary plaquette lying outside of vortex worldsheets (53).

\footnotetext{
${ }^{1}$ For the sake of completeness one may suggest to perform even more refined measurement by separating the contributions from the ultraviolet and the infrared vortex clusters following Ref. [37]. In our approach we have not done this refinement treating both the UV and IR vortices on the same footing.
} 
- In the low temperature region of the deconfinement phase $\left(T_{c}<T \lesssim 3 T_{c}\right)$ the specific vortex action is larger compared with its value at $T=0$.

- In all phases the specific vortex action is larger compared with the total action and, consequently, to the specific no-vortex action.

- The specific vortex action is very sensitive to the temperature variations compared with other action densities.

The last observation again indicates that the vortices play a very important role in the thermodynamics of Yang-Mills theory. We discuss this issue in the next Subsection.

\section{Center vortices and trace anomaly: numerical results}

The lattice calculation of the trace anomaly requires a careful renormalization. The renormalization of the total gluonic anomaly is quite straightforward (20): in order to cancel zero-point oscillations the expectation value of the $S U(2)$ plaquette calculated at zero-temperature should be subtracted from the finite-temperature plaquette action. According to a common wisdom, both these quantities contain the same UV divergencies, and the subtraction leaves us with the UV-finite thermal contribution ${ }^{2}$.

The regularization of the vortex contribution is less straightforward. According to Eq. (40) the gluonic action can be split into two pieces corresponding to the vortex contribution, $\left\langle S_{P}\right\rangle^{\text {vort }}$ and to the rest, $\left\langle S_{P}\right\rangle^{\text {novort }}$, both at zero and at finite temperature. However, the quantum fluctuations of the gluonic fields at the vortex and outside of vortex should not, in general, be the same because the vortices are infinitely thin objects possessing very strong gluonic fields [37]. Taking into account that the vortex density is a lively function of temperature, Figure 5. we conclude that the UV divergencies of, say, the action density $\left\langle S_{P}\right\rangle^{\text {vort }}$ at $T=0$ and $T>0$ should not, in general, match. Therefore, the renormalized finite-temperature value of $\left\langle S_{P}\right\rangle^{\text {novort }}$ by a subtraction of the same quantity at $T=0$ should give, in general, a UV-divergent result.

In order to illustrate a possible failure of a naive renormalization, we take the lattice expression for the trace anomaly (20), and separate the vortex contribution from the rest as follows:

$$
\begin{aligned}
\left\langle S_{P}\right\rangle_{T}- & \left\langle S_{P}\right\rangle_{0}=\left[\left\langle S_{P}\right\rangle_{T}^{\text {vort }}-\rho(T)\left\langle S_{P}\right\rangle_{0}\right] \\
& +\left\{\left\langle S_{P}\right\rangle_{T}^{\text {novort }}-[1-\rho(T)]\left\langle S_{P}\right\rangle_{0}\right\} .
\end{aligned}
$$

\footnotetext{
2 Note, however, the cautionary remark in Section IV B 1 about the renormalization of the vortex density: the naively "renormalized" vortex density turns out to be negative, Figure 5
}

Here we used Eq. (40) which implies that the vev of the action can exactly be split into the vortex contribution, $\left\langle S_{P}\right\rangle^{\text {vort }}$, and the contribution outside of vortices, $\left\langle S_{P}\right\rangle^{\text {novort}}$. This renormalization subtracts from the vortex-originated $\left\langle S_{P}\right\rangle_{T}^{\text {vort }}$ the appropriate amount of the $T=0$ gauge action, $\left\langle S_{P}\right\rangle_{0}$. The coefficient of proportionality is given by the vortex density $\rho$ : each vortex plaquette at $T>0$ is regularized by the plaquette action at $T=0$. The same type of the regularization is done for the no-vortex contribution, $\left\langle S_{P}\right\rangle^{\text {novort }}$.

According to Eqs. (20) and (54) the trace anomaly can then be split into the two regularized contributions

$$
\theta(T)=\theta_{\text {naive }}^{\text {vort }}(T)+\theta_{\text {naive }}^{\text {novort }}(T),
$$

where

$$
\begin{aligned}
\frac{\theta_{\text {naive }}^{\text {vort }}(T)}{T^{4}}= & 6 L_{t}^{4}\left(\frac{\partial \beta(a)}{\partial \log a}\right) \\
& \cdot\left[\left\langle S_{P}\right\rangle_{T}^{\text {vort }}-\rho(T)\left\langle S_{P}\right\rangle_{0}\right] \\
\frac{\theta_{\text {naive }}^{\text {novort }}(T)}{T^{4}}= & 6 L_{t}^{4}\left(\frac{\partial \beta(a)}{\partial \log a}\right) \\
& \cdot\left\{\left\langle S_{P}\right\rangle_{T}^{\text {novort }}-[1-\rho(T)]\left\langle S_{P}\right\rangle_{0}\right\}
\end{aligned}
$$

The results for the naively regularized quantities (56) and (57) are presented in Figure 10 (yet another simple regularization was implemented in our earlier investigation in Ref. [23]). We see from Figure 10 that the vortices provide a large negative contribution while the space outside of vortices is characterized by a large positive-valued contribution. The sum of the two gives the total trace anomaly. The naively regularized vortex and no-vortex results are not sensitive to the phase transition and they continue to rise as the temperature is lowered. Thus, we conclude the regularization schemes (56) and (57) are not appropriate.

It seems that the right quantity which characterizes the thermal contribution of the vortices to the action density is the specific vortex action (52), i.e. the vortex action per unit area of the vortex worldsheet. The regularization (i.e., the subtraction of the zero-point fluctuations at zero-temperature) should be done accordingly: the action per vortex plaquette at $T>0$ should be regularized by the action per vortex plaquette at $T=0$ :

$$
\left\langle s_{P}\right\rangle_{\mathrm{reg}}^{\text {vort }}=\left\langle s_{P}\right\rangle_{T}-\left\langle s_{P}\right\rangle_{0}
$$

where the specific vortex action $\left\langle s_{P}\right\rangle$ is defined in Eq. (52).

One can define the specific contribution $\vartheta^{\text {vort }}$ of the vortices into the trace anomaly, or, the contribution of the magnetic vortices to the trace anomaly counted per unit area of the vortex world surface at given temperature. Formally, the definition of this quantity is

$$
\vartheta^{\text {vort }}=\frac{\mathrm{d} \theta^{\text {vort }}}{\mathrm{d} \text { Area }},
$$

where "Area" denotes the area of the vortex worldsheet at given temperature. The definition of this quantity can 


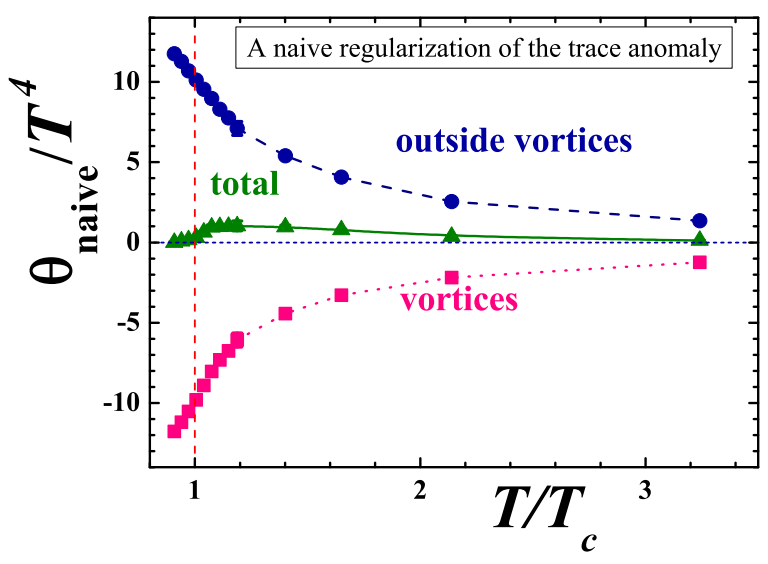

FIG. 10: (Color online) Naively regularized contribution of vortices into the trace anomaly (56) vs temperature (the data points are connected by the dotted line). The contribution to the anomaly coming from the space outside of vortices (57), and the total gluonic trace anomaly (20) are also shown (the dashed line and the solid line, respectively).

be written using Eqs. (20) and (58):

$$
\frac{\vartheta^{\mathrm{vort}}(T)}{T^{4}}=6 L_{t}^{4}\left(\frac{\partial \beta(a)}{\partial \log a}\right) \cdot\left(\left\langle s_{P}\right\rangle_{T}-\left\langle s_{P}\right\rangle_{0}\right) .
$$

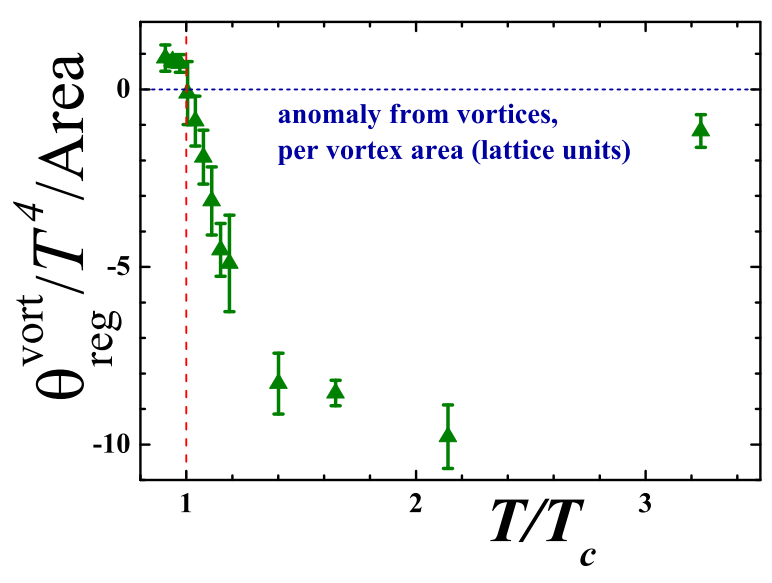

FIG. 11: (Color online) The specific anomaly, Eqs. (60) and (59), coming from the magnetic vortices (lattice units).

We show the specific contribution of the magnetic vortices to the trace anomaly in Figure 11 There are a few interesting features of this quantity: in the deconfinement phase $T_{c}<T \lesssim 3.5 T_{c}$ the specific anomaly is negative. In the deeper deconfinement phase as well as in the confinement phase the specific trace anomaly is positive. At the phase transition the specific anomaly is close to zero. All these features are in qualitative agreement with our preliminary calculations reported in Ref. [23].

The negative (ghostlike) value of the vortex-originated anomaly is not unexpected according to the theoretical analysis of Ref. [38]. The vortices can be treated as nonabelian strings with a nontrivial worldsheet dynamics associated, in particular, with the monopoles, localized on worldsheets of the magnetic vortices.

In physical units the specific vortex contribution to the anomaly is represented in Figure 12, The vortex contri-

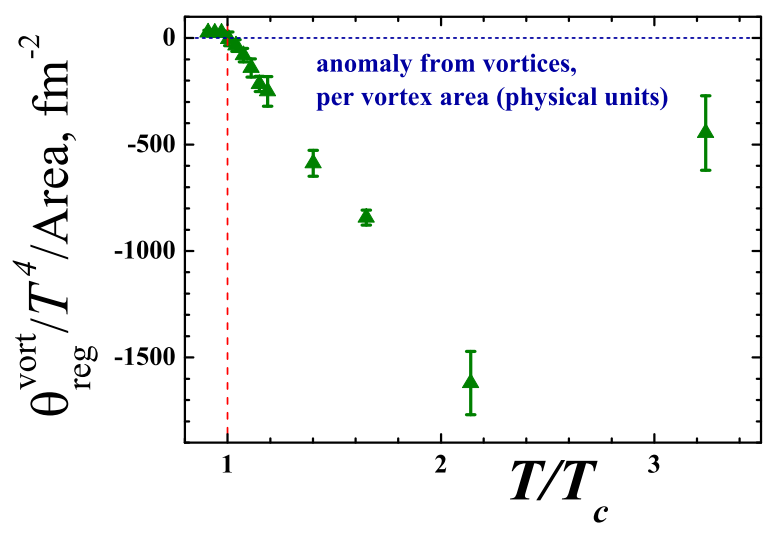

FIG. 12: (Color online) The same as in Fig. 11but in physical units.

bution is numerically very large. This fact agrees qualitatively with a singular nature of the distribution of the action density around vortex worldsheets [37].

Finally, the total regularized contribution of the vortices into the trace anomaly,

$$
\theta^{\mathrm{vort}}(T)=\rho(T) \vartheta^{\mathrm{vort}}(T),
$$

is represented in Figure 13. The scale of the vortexoriginated contribution is useful to compare with the total gluonic anomaly, Figure 2, Some features of the total

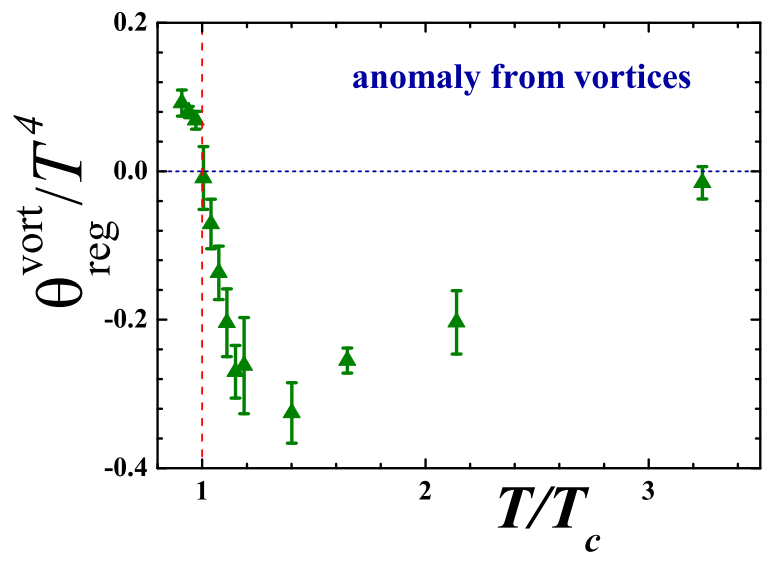

FIG. 13: (Color online) The contribution of the magnetic vortices into the trace anomaly, given by Eqs. (61) and (60).

vortex contribution to the anomaly (Figure 13) are very similar to the specific trace anomaly coming from vortices (Figure 12): in the confinement phase, $T<T_{c}$, the 
vortex trace anomaly is a positive quantity while in the deconfinement phase this quantity becomes negative in the region of temperatures $T_{c}<T \lesssim 3 T_{c}$. As for higher temperatures, $T \gtrsim 3 T_{c}$, our data does not allow us to discriminate between the two possibilities: the contribution to the trace anomaly coming from the vortices may be either positive or negative, and the ratio $\theta^{\text {vort }} / T^{4}$ in this region may be very small. Note that due to the definition of the vortex-originated anomaly, Eqs. (61) and (60), this quantity should be equal to zero at $T=0$. At the phase transition the anomaly is close to zero too.

It is appropriate to notice that at finite temperature some vortex trajectories may wrap with respect to the temporal (temperature) direction while other vortices may have zero wrapping index. Following Ref. [6] we suggest here that the wrapped vortices have a direct relation to the thermodynamics of the system while the unwrapped vortices bear features of the zero-temperature theory only. Thus we expect that the vortices with different wrappings with respect to the temporal direction may give different contributions to the gluonic anomaly. In our simulations we do not discriminate between the vortices with different wrapping properties.

Certain qualitative features of the vortex anomaly, Figure 13. may in principle be related to certain geometrical (orientational) properties of the vortex worldsheets. Indeed, it is well known [19, 21], that in the deep confinement phase, $T \ll T_{c}$, the worldsheets of the magnetic vortices have no preferred orientations in the lattice spacetime because there is practically no difference between spatial and temporal (temperature) directions. However, as the temperature increases the preferences do appear: the asymmetry ratio between the densities of the spacelike $\left({ }^{*} P_{i j}\right)$ and timelike $\left({ }^{*} P_{i 4}, i, j=1,2,3\right)$ vortex worldsheets slowly rises in the confinement phase up to a critical point [19, 21]. In the deconfinement phase the ratio falls down drastically since at $T \gg T_{c}$ the vortices are getting static according to the dimensional reduction arguments. On the other hand, we know that the chromomagnetic (spacelike, $\theta_{M} \sim \operatorname{Tr} U_{P_{i j}}$ ) part of the anomaly is always smaller than the chromoelectric (timelike, $\theta_{E} \sim \operatorname{Tr} U_{P_{i 4}}$ ) part, Figure 2. Since the spacelike vortices $\left({ }^{*} P_{i j}\right)$ pierce timelike $\left(P_{i 4}\right)$ plaquettes and vise versa, the slow rise of the spacelike portion of the vortex worldsheets in the confinement phase leads to an increase of the rôle of the timelike plaquettes in the vortex-dominated anomaly.

The timelike (chromoelectric) part of the bulk anomaly is bigger than the chromomagnetic part according to Figure 2, If the same is true for the anomaly at the vortex worldsheet then the gradual orientation of the magnetic vortices towards spatial directions with the rise of temperature should lead to an increase of the vortex-originated anomaly in accordance with our observations (Figure 13). As the system passes the deconfining point, the timelike vortices become more favored compared with the spacelike ones and therefore the spacelike chromomagnetic plaquettes become domi- nant in the vortex-originated contribution. Thus, taking into account the relation $\theta_{M}<\theta_{E}$ and using the geometrical arguments, one can qualitatively explain a drop of the vortex-originated anomaly just above the critical temperature, Figure 13.

It is also important to stress that just above the phase transition the trace anomaly coming from the vortices (Figure 13) is approximately of the same order as the total gluon anomaly (Figure 2). This fact highlights the significance of the vortex degrees of freedom to the thermodynamical behavior of the gluon plasma.

Our numerical calculations do not allow us to trace the behavior of the vortex-originated trace anomaly in the confinement phase at low enough temperatures, $T<$ $0.8 T_{c}$. As this quantity is still not zero at low temperatures, we cannot calculate the contribution of the vortices into the pressure (12) and into the energy density (13) because they involve integration over the low temperature region. More refined and accurate calculations are needed to address this issue properly.

A qualitative extrapolation of our data into the lowtemperature domain of the phase diagram allows us to suggest the behavior of the vortex contribution to the trace anomaly as it is visualized in Figure 14.

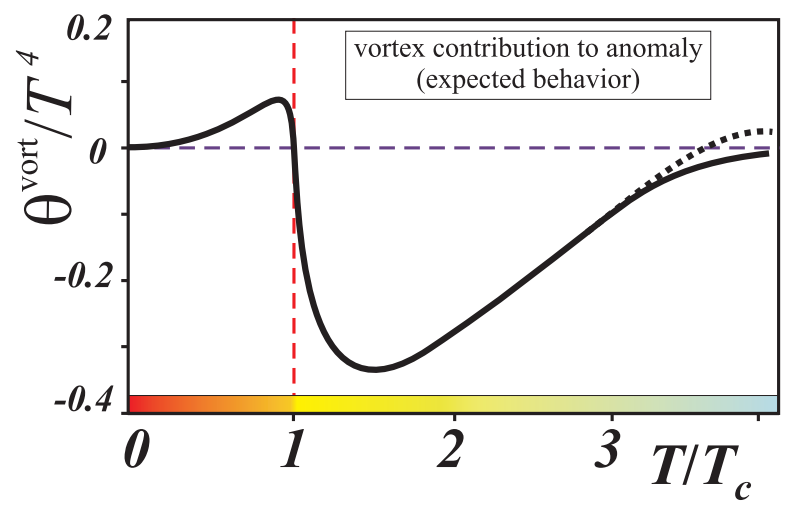

FIG. 14: (Color online) An expected behavior of the contribution of the magnetic vortices into the gluonic trace anomaly (the solid line). The dotted line represents another hightemperature behavior that cannot be excluded by the available data.

\section{SUMMARY OF NUMERICAL RESULTS}

We observe the intriguing relevance of magnetic vortices to the thermodynamics of Yang-Mills theory. A brief summary of the vortex properties is as follows:

\section{Vortex density, Figure 5}

- $T<T_{c}$. In the cold region of the confinement phase $\rho(T)$ is an increasing function of temperature $T$. 
As the temperature gets higher, the increase of the density turns into a sharp fall.

- $T=T_{c}$. At the phase transition the vortex density is close to its zero temperature value, $\rho\left(T_{c}\right) \approx \rho(0)$.

- $T_{c}<T \lesssim 2 T_{c}$. Right above the critical temperature the vortex density continues to fall and the minimum of the vortex density is reached approximately around $T \approx 2 T_{c}$. At this temperature the magnetic contribution to the trace anomaly vanishes, Figure 2 .

- $2 T_{c} \lesssim T \lesssim 3 T_{c}$. The vortex density start to rise, and at $T \approx 3 T_{c}$ the density reaches its zerotemperature value again, $\rho\left(3 T_{c}\right) \approx \rho(0)$.

- $T \gtrsim 3 T_{c}$. In this domain of the temperatures the density is a positively defined rising quantity.

- $T \gg T_{c}$. At very high temperatures the vortex density should follow the law of Eq. (50).

Our results on the vortex properties are in a qualitative agreement with other studies of the vortex properties at finite temperature [19, 20, 21, 22]. The rise and fall of the vortex density with the increase of temperature in the confinement phase should be understood as a result of the competition of two opposite processes: the thermal fluctuations (i) excite the vortex media leading to creation of additional virtual vortex loops from the vacuum; (ii) destroy the chaotic percolation of the vortex cluster in the temporal direction making the vortex trajectories more static. The percolation of the vortex trajectories in the temporal direction is not a well defined quantity because of the finiteness of the extent of the Euclidean spacetime in this direction. Nevertheless, at low temperatures not all the vortex loops wind around this direction and the increase of the temperature tries to break the loops into pieces.

\section{Vortex contribution into the trace anomaly, Figures 13 and 14}

We found that vortices do contribute to the equation of state of the gluon plasma. The vortex contribution is illustrated in Figures 13 and 14. Qualitatively, the temperature behavior of the vortex trace anomaly is very similar to the behavior of the vortex density described above. We observe that:

- in the confinement phase the vortex trace anomaly is positively defined,

- at the critical temperature, $T=T_{c}$, the vortexoriginated trace anomaly is consistent with zero,

- in the low-temperature domain of the deconfinement phase $\left(T_{c}<T \lesssim 3.5 T_{c}\right)$ the trace anomaly coming from the vortices is negative,
- at the high-temperature region of the deconfinement phase $\left(T \gtrsim 3.5 T_{c}\right)$ the contribution of the vortices to the gluon trace anomaly may either become positive or stay negative. Anyway, the ratio $\theta^{\text {vort }}(T) / T^{4}$ is expected to be small in this region,

- qualitatively, the features of the specific vortex trace anomaly (Figures 11] and 12) and the "bulk" vortex trace anomaly (Figure [13) are very similar to each other,

- the gluon action density at the vortex trajectories (Figure 9) is much higher compared with the gluon action density outside of vortex worldsheets. This result is an agreement with previous studies reported in Ref. [37], and

- the value of the gluon action density at the vortices is much more sensitive to the temperature variations compared with the gluon action density outside of vortices (Figure 9]).

One can characterize the very strong contribution of the vortices into the trace anomaly and, consequently, to the equation of the state of the Yang-Mills plasma as follows: on our lattices the vortices occupy just 2-4\% of the lattice spacetime (Figure 4) in the deconfinement phase while their regularized contribution to the trace anomaly (Figure 13) is of the order of the total contribution of all gluons in the bulk of the system (Figure 2).

\section{CONCLUSIONS}

The basic idea of this paper is to calculate the contribution of vortices to the vev of the Euclidean action density of the gluonic fields. The action density, which is basically the gluon condensate, is related to the thermodynamic quantities of the Yang-Mills theory - such as pressure and energy density - via the anomaly of the trace of the energy-momentum tensor. A short summary of the results is presented in the previous Section.

We observed that magnetic vortices play an essential rôle in the thermodynamics of Yang-Mills theory. Our main result is presented in Figure 13. In the temperature range $T_{c}<T \lesssim 3 T_{c}$ the contribution of the magnetic vortex strings to the trace anomaly is numerically large and negative in sign compared to the total energy and the trace of the energy-momentum tensor of the whole of the plasma:

$$
\theta_{\text {vort }}(T)<0, \quad\left\langle\operatorname{Tr} G^{2}(T)\right\rangle_{\text {vort }}>0, \quad T_{c}<T \lesssim 3 T_{c} .
$$

Taken at face value, Eqs. (62) amount to the observation of a ghostlike component of the gluonic plasma.

The existence of magnetic vortices may strongly influence the physics of quarks in the quark-gluon plasma. The random chromomagnetic fluxes of the string networks in the gluon plasma may trap, scatter and drag the quarks. The chromoelectric and chromomagnetic flux 
tubes are suggested to arise in the early Glasma regime of the evolution of color gauge fields in high energy heavy ion collisions [39]. We leave to a future publication [40] discussions of the magnetic string effects in thermally excited gluon media.

There are, however, reservations to be made. First, the theoretical interpretation of data referring to a component of the plasma is subject to an uncertainty because the magnetic component obviously interacts strongly with the rest of the plasma. And it is rather the total energy that has a direct physical meaning than the distribution of it. Thus, a detailed interpretation of the data (62) asks for further efforts on the continuum-theory side. Second, it might be worth emphasizing that, observationally, the negative sign is due to a decrease of the density of the strings (compared with the $T<T_{c}$ case). At this moment, it is not clear whether this mechanism, behind the signs in Eq.(62) is important for the interpretation.

In any case, the numerical calculations reveal that magnetic strings, which at our lattices occupy (2-4)\% of the total volume are crucial for the dynamics of the whole plasma (in the limit of the vanishing lattice spacing $a \rightarrow 0$ this fraction tends to zero). The absolute value of their contribution to the energy of the plasma is of the order of the total value of the energy in the bulk of the system. Thus, perturbative calculations, like (1), agree with the data averaged over the whole plasma while nonperturbative dynamics is responsible for a highly nontrivial sharing of the same energy density between various components of the plasma. A remote analogy is a quark- resonance duality. While simple quark graphs describe well the cross section averaged over a large energy interval, resonances are responsible for a more local-in-energy structure. It is becoming obvious that a detailed understanding of the plasma properties is not possible without clarifying the role of its magnetic component [6].

\section{Acknowledgments}

This work was supported by Grants-in-Aid for Scientific Research from "The Ministry of Education, Culture, Sports, Science and Technology of Japan" Nos. 17340080 and 20340055 , by the STINT Institutional grant IG20042 025, by the grants RFBR 06-02-04010-NNIO-a, RFBR 08-02-00661-a, DFG-RFBR 436 RUS, by a grant for scientific schools NSh-679.2008.2, by the Federal Program of the Russian Ministry of Industry, Science and Technology No. 40.052.1.1.1112 and by the Russian Federal Agency for Nuclear Power. The numerical simulations were performed using a SX-8 supercomputer at RCNP at Osaka University, and a SR11000 machine at Hiroshima University. M.N.Ch is thankful to the members of the Department of Theoretical Physics of Uppsala University for kind hospitality and stimulating environment. M.N.Ch and V.I.Z appreciate their fruitful stays at the Institute for Theoretical Physics of Kanazawa University and the Research Institute for Information Science and Education of Hiroshima University, Japan.
[1] E. V. Shuryak, in "Minneapolis 2006, Continuous advances in QCD", p. 3, Ed. by M. Peloso and M. Shifman (Singapore, World Scientific, 2007) hep-ph/0608177); J. P. Blaizot, J. Phys. G 34, S243 (2007) hep-ph/0703150.

[2] F. Karsch, Nucl. Phys. A 783, 13 (2007) hep-ph/0610024; U. M. Heller, PoS LAT2006, 011 (2006) hep-lat/0610114.

[3] J. J. Friess, S. S. Gubser, G. Michalogiorgakis, S. S. Pufu, JHEP 0704, 080 (2007) hep-th/0611005.

[4] D. H. Rischke, M. I. Gorenstein, A. Schafer, H. Stoecker, W. Greiner, Phys. Lett. B 278 (1992) 19; A. Peshier, B. Kampfer, O. P. Pavlenko, G. Soff, Phys. Rev. D 54, 2399 (1996); P. Levai, U. W. Heinz, Phys. Rev. C 57, 1879 (1998) arXiv:hep-ph/9710463; V. M. Bannur, arXiv:0807.2092.

[5] D. Teaney, Phys. Rev. C 68, 034913 (2003) arXiv:nucl-th/0301099; A. Nakamura, S. Sakai, Phys. Rev. Lett. 94, 072305 (2005) hep-lat/0406009; H. B. Meyer, Phys. Rev. D 76, 101701(R) (2007) arXiv:0704.1801.

[6] M. N. Chernodub, V. I. Zakharov, Phys. Rev. Lett. 98, 082002 (2007) hep-ph/0611228.

[7] J. Liao, E. Shuryak, Phys. Rev. C 75, 054907 (2007) hep-ph/0611131.

[8] P. Giovannangeli, C. P. Korthals Altes, Nucl. Phys.
B608, 203 (2001) hep-ph/0102022; C. P. Korthals Altes, in "Minneapolis 2006, Continuous advances in QCD", p. 26 hep-ph/0607154; M. Baker, Phys. Rev. D 78, 014009 (2008) arXiv:0711.4861 [hep-ph]].

[9] J. Greensite, Prog. Part. Nucl. Phys. 51, 1 (2003) hep-lat/0301023.

[10] Y. Nambu, Phys. Rev. D 10, 4262 (1974); G. 't Hooft, in High Energy Physics, ed. A. Zichichi, EPS International Conference, Palermo (1975); S. Mandelstam, Phys. Rept. 23, 245 (1976); T. Suzuki and I. Yotsuyanagi, Phys. Rev. D 42, 4257 (1990).

[11] M. N. Chernodub, M. I. Polikarpov, in "Confinement, duality, and nonperturbative aspects of QCD", Ed. by Pierre Van Baal (New York, Plenum Press, 1998), hep-th/9710205 M. N. Chernodub, F. V. Gubarev, M. I. Polikarpov, A. I. Veselov, Prog. Theor. Phys. Suppl. 131, 309 (1998) arXiv:hep-lat/9802036.

[12] J. Ambjorn, J. Giedt, J. Greensite, JHEP 0002, 033 (2000) hep-lat/9907021]; V. I. Zakharov, AIP Conf. Proc. 756, 182 (2005) hep-ph/0501011.

[13] J. M. Cornwall, Phys. Rev. D 58, 105028 (1998) arXiv:hep-th/9806007.

[14] B. L. G. Bakker, A. I. Veselov, M. A. Zubkov, Phys. Lett. B 471, 214 (1999) arXiv:hep-lat/9902010.

[15] M. A. C. Kneipp, Phys. Rev. D 68, 045009 (2003) hep-th/0211049; Phys. Rev. D 69, 045007 (2004) 
arXiv:hep-th/0308086; R. Auzzi, S. Bolognesi, J. Evslin, K. Konishi, Nucl. Phys. B686, 119 (2004) hep-th/0312233; D. Tong, Phys. Rev. D 69, 065003 (2004) hep-th/0307302; A. Gorsky, M. Shifman, A. Yung, ibid. 71, 045010 (2005) hep-th/0412082; Y. Isozumi, M. Nitta, K. Ohashi and N. Sakai, Phys. Rev. D 71, 065018 (2005) arXiv:hep-th/0405129; M. Eto, Y. Isozumi, M. Nitta, K. Ohashi and N. Sakai, Phys. Rev. D 72, 025011 (2005) arXiv:hep-th/0412048; J. Phys. A 39, R315 (2006) arXiv:hep-th/0602170; M. Shifman, A. Yung, Rev. Mod. Phys. 79, 1139 (2007) hep-th/0703267.

[16] M.N. Chernodub, R. Feldmann, E.-M. Ilgenfritz, A. Schiller, Phys. Lett. B605, 161 (2005) hep-lat/0406015.

[17] M. N. Chernodub, V. I. Zakharov, "Monopoles and vortices in Yang-Mills plasma", to appear in Phys. Atom. Nucl. arXiv:0806.2874.

[18] V. I. Zakharov, Braz. J. Phys. 37, 165 (2007) hep-ph/0612342 ; AIP Conf. Proc. 756, 182 (2005) hep-ph/0501011.

[19] K. Langfeld, O. Tennert, M. Engelhardt, H. Reinhardt, Phys. Lett. B 452, 301 (1999) hep-lat/9805002.

[20] M. N. Chernodub, M. I. Polikarpov, A. I. Veselov, M. A. Zubkov, Nucl. Phys. Proc. Suppl. 73, 575 (1999) hep-lat/9809158.

[21] R. Bertle, M. Faber, J. Greensite, S. Olejnik, JHEP 9903, 019 (1999) arXiv:hep-lat/9903023.

[22] M. Engelhardt, K. Langfeld, H. Reinhardt, O. Tennert, Phys. Rev. D 61, 054504 (2000) hep-lat/9904004.

[23] M. N. Chernodub, K. Ishiguro, A. Nakamura, T. Sekido, T. Suzuki, V. I. Zakharov, PoS LAT2007, 174 (2007) arXiv:0710.2547.

[24] J. Engels, F. Karsch, H. Satz, I. Montvay, Nucl. Phys. B 205, 545 (1982); J. Engels, J. Fingberg, K. Redlich, H. Satz, M. Weber, Z. Phys. C 42, 341 (1989); J. Engels, J. Fingberg, F. Karsch, D. Miller, M. Weber, Phys. Lett. B 252, 625 (1990); J. Engels, F. Karsch, K. Redlich, Nucl. Phys. B 435, 295 (1995) hep-lat/9408009.

[25] G. Boyd, J. Engels, F. Karsch, E. Laermann, C. Lege- land, M. Lutgemeier, B. Petersson, Phys. Rev. Lett. 75, 4169 (1995) hep-lat/9506025; Nucl. Phys. B 469, 419 (1996) hep-lat/9602007.

[26] J. Engels, J. Fingberg, M. Weber, Nucl. Phys. B 332, 737 (1990).

[27] J. Fingberg, U. M. Heller, F. Karsch, Nucl. Phys. B 392, 493 (1993) hep-lat/9208012; M. J. Teper, hep-th/9812187.

[28] J. C. R. Bloch, A. Cucchieri, K. Langfeld, T. Mendes, Nucl. Phys. B 687, 76 (2004) hep-lat/0312036.

[29] M. N. Chernodub and E. M. Ilgenfritz, Phys. Rev. D 78, 034036 (2008) arXiv:0805.3714 [hep-lat]].

[30] P. de Forcrand, M. D'Elia, Phys. Rev. Lett. 82, 4582 (1999) hep-lat/9901020.

[31] L. Del Debbio, M. Faber, J. Greensite, S. Olejnik, Phys. Rev. D 55, 2298 (1997) hep-lat/9610005; L. Del Debbio, M. Faber, J. Giedt, J. Greensite, S. Olejnik, Phys. Rev. D 58, 094501 (1998) hep-lat/9801027.

[32] V. G. Bornyakov, D. A. Komarov, M. I. Polikarpov, Phys. Lett. B 497, 151 (2001) hep-lat/0009035.

[33] J. I. Kapusta, "Finite-Temperature Field Theory" (Cambridge University Press, Cambridge, 1989).

[34] A. D'Alessandro, M. D'Elia, Nucl. Phys. B799, 241 (2008) arXiv:0711.1266.

[35] J. Liao, E. Shuryak, arXiv:0804.0255

[36] M. N. Chernodub, K. Ishiguro, T. Suzuki, JHEP 0309 027, (2003) hep-lat/0204003.

[37] F. V. Gubarev, A. V. Kovalenko, M. I. Polikarpov, S. N. Syritsyn, V. I. Zakharov, Phys. Lett. B 574, 136 (2003) hep-lat/0212003; A. V. Kovalenko, M. I. Polikarpov, S. N. Syritsyn, V. I. Zakharov, Phys. Rev. D 71, 054511 (2005) hep-lat/0402017.

[38] A. Gorsky, V. Zakharov, Phys. Rev. D 77, 045017 (2008) arXiv:0707.1284.

[39] L. McLerran, arXiv:0807.4095 A. Dumitru, F. Gelis, L. McLerran and R. Venugopalan, Nucl. Phys. A 810, 91 (2008) arXiv:0804.3858 [hep-ph]].

[40] M.N.Chernodub, V.I.Zakharov, in preparation. 